\title{
Plasmonic nanostars as efficient broadband scatterers for random lasing
}

Johannes Ziegler, * Christian Wörister, Cynthia Vidal, Calin Hrelescu, and Thomas A. Klar

Institute of Applied Physics, Johannes Kepler University Linz, 4040 Linz, Austria

\section{Supporting Information}

\section{Cross sections from numerical calculations of a gold nanostar and a dielectric silicon dioxide nanoparticle}

Metallic nanoparticles feature huge field enhancements due to plasmon resonances, but also exhibit absorption losses, which might be detrimental to the random lasing performance. We conducted numerical calculations in order to estimate the scattering and absorption cross sections of an exemplary gold nanostar and a dielectric sphere of comparable size.

The scattering (Figure S1, red curve) and absorption (Figure S1, blue curve) cross sections of a representative nanostar (Inset in Figure S1) were calculated using the commercial software FDTD Solutions (version 8.9.269) from Lumerical Solutions Inc., which provides a 3D Maxwell equation solver based on Finite-Difference Time-Domain method (FDTD). The simulated nanostar consists of a spherical core (diameter $80 \mathrm{~nm}$ ) and 10 tips of $70 \mathrm{~nm}$ length protruding out of the core, modeled as rounded tip cones ( $80 \mathrm{~nm}$ height, tip radius $8 \mathrm{~nm}$ and $30^{\circ}$ cone angle). In the modeling, the permittivity of gold was obtained by a generalized multi-coefficient fit of the permittivity of bulk gold according to Johnson and Christy. ${ }^{\text {S1 }}$ A background refractive index of 1.33 for water was used. The Total Field/Scattered Field (TFSF) source implemented in the software was used as excitation source. The cross sections of a silicon dioxide $\left(\mathrm{SiO}_{2}\right)$ sphere with $160 \mathrm{~nm}$ diameter in water were calculated using Mie theory. Since $\mathrm{SiO}_{2}$ has negligible absorption in the calculated wavelength range, the displayed extinction cross section (Figure S1, black curve 100x magnified) also corresponds to the scattering cross section.

The scattering cross section of the dielectric $\mathrm{SiO}_{2}$-sphere is about two orders of magnitude lower than that of a plasmonic nanostar. The non-resonant Mie-type scattering of the dielectric sphere rapidly reduces towards longer wavelengths, whereas the nanostar with its plasmon resonances provides dramatically higher scattering cross sections in the near-infrared spectral region. 


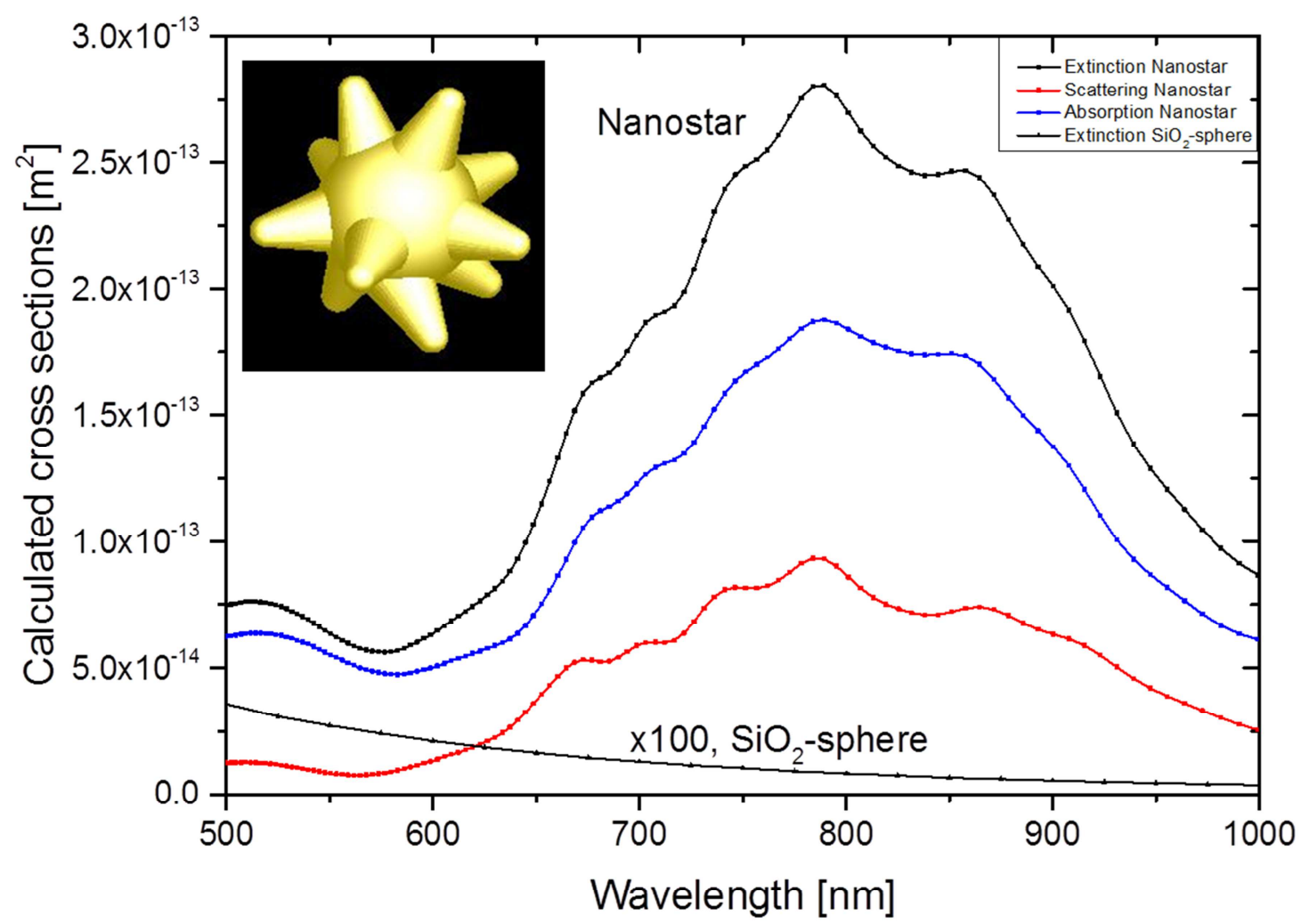

Figure S1. Calculated cross sections of a gold nanostar and a silicon dioxide sphere of $160 \mathrm{~nm}$ diameter. Inset: Geometry of the nanostar used for the calculations.

\section{Emission spectra of random lasers containing plasmonic nanostars and random lasers containing dielectric nanoparticles at various pump fluences}

We performed additional experiments to directly compare the random lasing behavior of gold nanostars as scattering centers to that of the dielectric nanoparticles. The ensemble extinction of spherical $\mathrm{SiO}_{2}$-nanoparticles (MicroSil Microspheres, Bangs Laboratories, Inc., mean diameter $0.16 \mu \mathrm{m}$ ) was measured and compared to the calculated extinction cross sections in order to adjust a defined nanoparticle density of $2.3 \times 10^{9} \mathrm{~cm}^{-3}$ in the random laser. Likewise, the nanostar density was estimated to a comparable, yet slightly lower density of $1 \times 10^{9} \mathrm{~cm}^{-3}$. For the three gain media Rhodamine 6G, Styryl 15 and Styryl 14, covering the broad wavelength range from $560 \mathrm{~nm}$ to $970 \mathrm{~nm}$, we prepared solutions containing either nanostars or $\mathrm{SiO}_{2}$-spheres.

The emission spectra of the random lasers are presented in Figure S2 for Rhodamine 6G, in Figure S3 for Styryl 15 and in Figure S4 for Styryl 14. The left panels correspond to random lasers containing nanostars, whereas the right panels correspond to those with $\mathrm{SiO}_{2}$-spheres. 
Each segment includes 50 spectra for consecutive, single excitation pulses at the indicated pump fluence. Emission intensities above 64000 are truncated due to saturation of the CCD.

It can be clearly observed for every gain medium from the evolution of the emission with increasing pump fluences that the random lasers comprising the gold nanostars exhibit characteristic lasing peaks above threshold. At the same pump fluences, the random lasers comprising $\mathrm{SiO}_{2}$-spheres only show ASE background for Rhodamine 6G and Styryl 15 and only weak spectrally narrow peaks at the highest pump fluence for Styryl 14. The random lasing performance of plasmonic nanostars as scattering centers is, despite existing absorption losses, obviously superior to that of $\mathrm{SiO}_{2}$-spheres at comparable particle densities.

\section{References}

(S1) Johnson, P. B.; Christy, R. W. Optical Constants of the Noble Metals. Phys. Rev. B 1972, $6(12), 4370-4379$. 

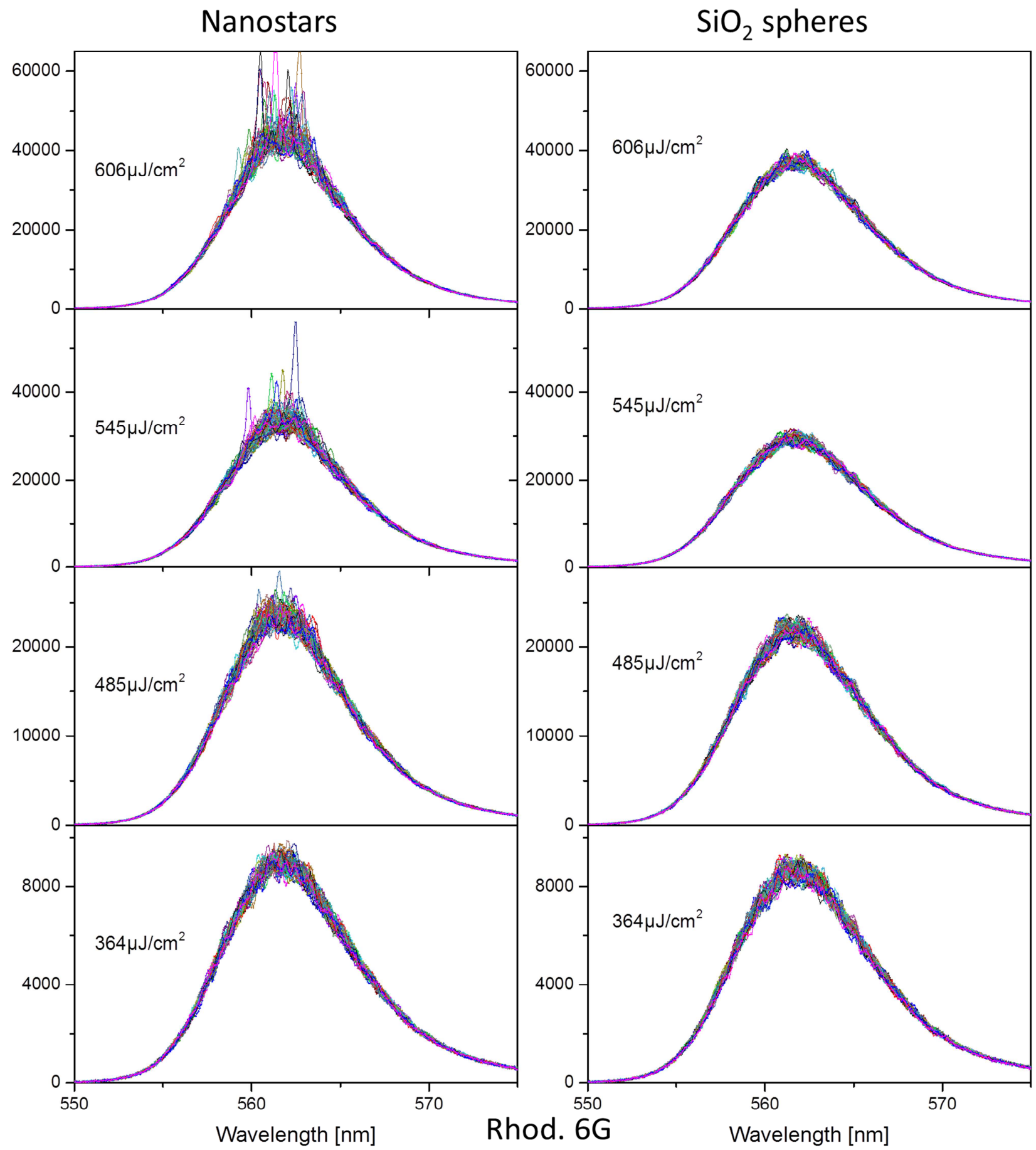

Figure S2. Emission spectra of a random laser containing Rhodamine $6 \mathrm{G}$ as gain medium and either gold nanostars (left panel) or silicon dioxide spheres (right panel) at similar particle densities. Each segment includes 50 spectra for consecutive excitation pulses at the indicated pump fluence. 


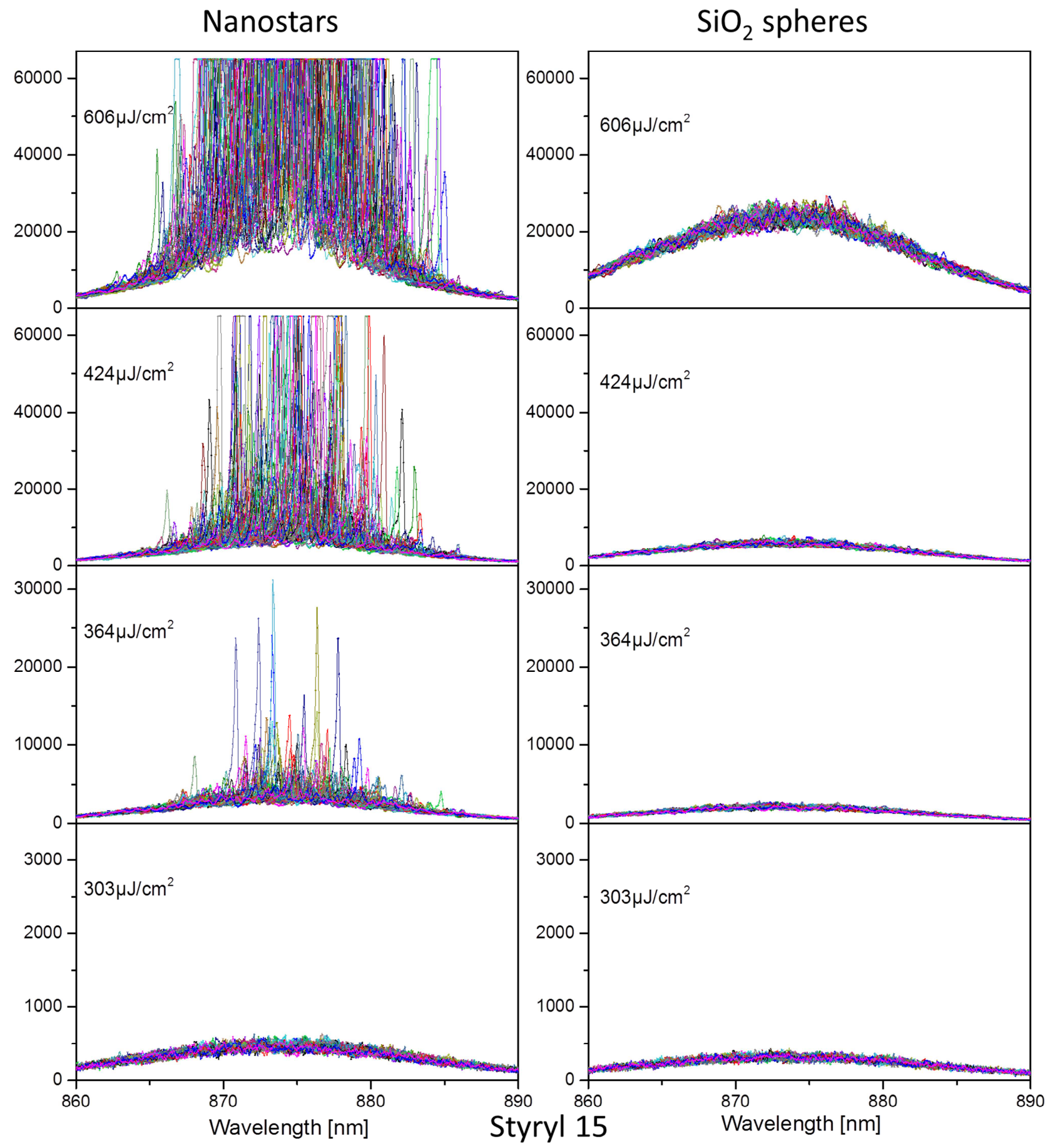

Figure S3. Emission spectra of a random laser containing Styryl 15 as gain medium and either gold nanostars (left panel) or silicon dioxide spheres (right panel) at similar particle densities. Each segment includes 50 spectra for consecutive excitation pulses at the indicated pump fluence. 


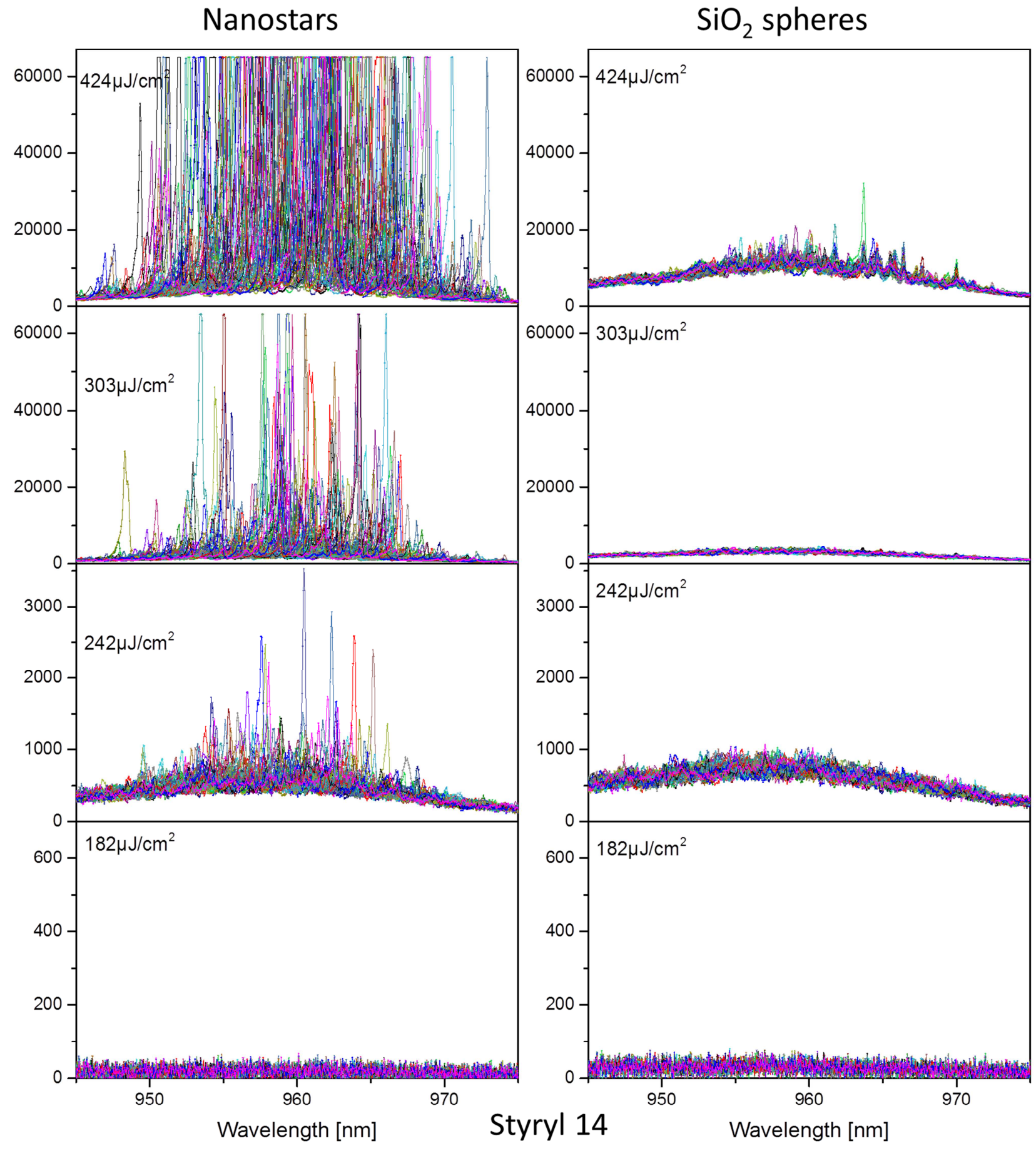

Figure S4. Emission spectra of a random laser containing Styryl 14 as gain medium and either gold nanostars (left panel) or silicon dioxide spheres (right panel) at similar particle densities. Each segment includes 50 spectra for consecutive excitation pulses at the indicated pump fluence. 